\title{
HARDY-TYPE INEQUALITIES FOR INTEGRAL TRANSFORMS ASSOCIATED WITH JACOBI OPERATOR
}

\author{
M. DZIRI AND L. T. RACHDI
}

Received 8 April 2004 and in revised form 24 December 2004

We establish Hardy-type inequalities for the Riemann-Liouville and Weyl transforms associated with the Jacobi operator by using Hardy-type inequalities for a class of integral operators.

\section{Introduction}

It is well known that the Jacobi second-order differential operator is defined on $] 0,+\infty$ [ by

$$
\Delta_{\alpha, \beta} u(x)=\frac{1}{A_{\alpha, \beta}(x)} \frac{d}{d x}\left(A_{\alpha, \beta}(x) \frac{d u}{d x}\right)+\rho^{2} u(x),
$$

where

(i) $A_{\alpha, \beta}(x)=2^{2 \rho} \sinh ^{2 \alpha+1}(x) \cosh ^{2 \beta+1}(x)$,

(ii) $\alpha, \beta \in \mathbb{R} ; \alpha \geq \beta>-1 / 2$,

(iii) $\rho=\alpha+\beta+1$.

The Riemann-Liouville and Weyl transforms associated with Jacobi operator $\Delta_{\alpha, \beta}$ are, respectively, defined, for every nonnegative measurable function $f$, by

$$
\begin{aligned}
R_{\alpha, \beta}(f)(x) & =\int_{0}^{x} k_{\alpha, \beta}(x, y) f(y) d y, \\
W_{\alpha, \beta}(f)(y) & =\int_{y}^{\infty} k_{\alpha, \beta}(x, y) f(x) A_{\alpha, \beta}(x) d x,
\end{aligned}
$$

where $k_{\alpha, \beta}$ is the nonnegative kernel defined, for $x>y>0$, by

$$
\begin{aligned}
k_{\alpha, \beta}(x, y)= & \frac{2^{-\alpha+3 / 2} \Gamma(\alpha+1)(\cosh (2 x)-\cosh (2 y))^{\alpha-1 / 2}}{\sqrt{\pi} \Gamma(\alpha+1 / 2) \cosh ^{\alpha+\beta}(x) \sinh ^{2 \alpha}(x)} \\
& \times F\left(\alpha+\beta, \alpha-\beta ; \alpha+\frac{1}{2} ; \frac{\cosh (x)-\cosh (y)}{2 \cosh (x)}\right)
\end{aligned}
$$


and $F$ is the Gaussian hypergeometric function. Such integral transforms have many applications to science and engineering $[3,4]$.

These operators have been studied on regular spaces of functions. In particular in [19], the author has proved that the Riemann-Liouville transform $R_{\alpha, \beta}$ is an isomorphism from $\xi_{*}(\mathbb{R})$ (the space of even infinitely differentiable functions on $\mathbb{R}$ ) on itself, and that the Weyl transform $W_{\alpha, \beta}$ is an isomorphism from $D_{*}(\mathbb{R})$ (the space of even infinitely differentiable functions on $\mathbb{R}$ with compact support) on itself. The Weyl transform has also been studied on Shwartz space $S_{*}(\mathbb{R})[20]$.

This paper is devoted to the study of the Riemann-Liouville and Weyl transforms on the spaces

$$
L^{p}\left(\left[0, \infty\left[, A_{\alpha, \beta}(x) d x\right) \quad 1<p<\infty\right.\right.
$$

of measurable functions on $[0, \infty[$ such that

$$
\|f\|_{p, \alpha, \beta}=\left(\int_{0}^{\infty}|f(x)|^{p} A_{\alpha, \beta}(x) d x\right)^{1 / p}<\infty .
$$

The main results of this work are Theorems 4.2 and 4.4 in Section 4.

To obtain those results we use the following integral operators:

$$
\begin{aligned}
& T_{\varphi}(f)(x)=\int_{0}^{x} \varphi\left(\frac{t}{x}\right) f(t) \nu(t) d t, \\
& T_{\varphi}^{*}(g)(x)=\int_{x}^{\infty} \varphi\left(\frac{x}{t}\right) g(t) d \mu(t),
\end{aligned}
$$

where

(i) $v$ is a nonnegative locally integrable function on $[0, \infty[$,

(ii) $d \mu(t)$ is a nonnegative measure, locally finite on $[0, \infty[$,

(iii) the following is a measurable function satisfying some properties $[10,12,18]$ :

$$
\varphi:] 0,1[\longrightarrow] 0, \infty[
$$

Both operators $T_{\varphi}$ and $T_{\varphi}^{*}$ are connected by the following duality relation: for all nonnegative measurable functions $f$ and $g$ we have

$$
\int_{0}^{\infty} T_{\varphi}(f)(x) g(x) d \mu(x)=\int_{0}^{\infty} f(y) T_{\varphi}^{*}(g)(y) \nu(y) d y
$$

In this paper, we give some conditions on the functions $\varphi, \nu$ and the measure $d \mu$ so that the operator $T_{\varphi}$ and its dual $T_{\varphi}^{*}$ satisfy the following Hardy inequalities: for all real numbers $p, q$ satisfying

$$
1<p \leq q<\infty
$$


there exists a positive constant $C_{p, q}$ such that for all nonnegative measurable functions $f$ and $g$ we have

$$
\begin{gathered}
\left(\int_{0}^{\infty}\left(T_{\varphi}(f)(x)\right)^{q} d \mu(x)\right)^{1 / q} \leq C_{p, q}\left(\int_{0}^{\infty}(f(x))^{p} \nu(x) d x\right)^{1 / p}, \\
\left(\int_{0}^{\infty}\left(T_{\varphi}^{*}(g)(x)\right)^{p^{\prime}} \nu(x) d x\right)^{1 / p^{\prime}} \leq C_{p, q}\left(\int_{0}^{\infty}(g(x))^{q^{\prime}} d \mu(x)\right)^{1 / q^{\prime}},
\end{gathered}
$$

where $p^{\prime}$ and $q^{\prime}$ are the conjugate exponents, respectively, of $p$ and $q$.

In [5], we have studied inequalities (1.10), in the case $1<q<p<\infty$. The inequalities obtained below for the operators $T_{\varphi}$ and $T_{\varphi}^{*}$ will allow us to obtain the main results of this paper.

This paper is arranged as follows.

In Section 2, we consider a continuous nonincreasing function

$$
\varphi:] 0,1[\longrightarrow] 0, \infty[
$$

for which there exists a positive constant $D$ satisfying

$$
\forall x, y \in] 0,1[, \quad \varphi(x y) \leq D(\varphi(x)+\varphi(y)) .
$$

Then we give necessary and sufficient conditions such that the operators $T_{\varphi}$ and $T_{\varphi}^{*}$ satisfy the inequalities (1.10).

In Section 3, we suppose only that the function $\varphi$ is nondecreasing and we give the sufficient conditions such that the precedent inequalities hold.

In Section 4, we use the results obtained below to study and to establish the Hardy inequalities for Riemann and Weyl operators associated with Jacobi differential operator $\Delta_{\alpha, \beta}$.

\section{Hardy operator $T_{\varphi}$ and its dual $T_{\varphi}^{*}$ when the function $\varphi$ is nonincreasing on ]0,1[}

In this section, we consider a measurable positive and nonincreasing function $\varphi$ defined on ]0,1 [ for which we associate the operator $T_{\varphi}$ and its dual $T_{\varphi}^{*}$ defined, respectively, for every nonnegative and measurable function $f$, by

$$
\begin{aligned}
& \forall x>0, \quad T_{\varphi}(f)(x)=\int_{0}^{x} \varphi\left(\frac{t}{x}\right) f(t) \nu(t) d t, \\
& \forall x>0, \quad T_{\varphi}^{*}(f)(x)=\int_{x}^{\infty} \varphi\left(\frac{x}{t}\right) f(t) d \mu(t),
\end{aligned}
$$

where $v$ is a measurable nonnegative function on $] 0, \infty[$ such that

$$
\forall a>0, \quad \int_{0}^{a} v(t) d t<\infty
$$

and $d \mu(t)$ is a nonnegative measure on $[0, \infty[$ satisfying

$$
\forall 0<a<b, \quad \int_{a}^{b} d \mu(t)<\infty .
$$


The main result of this section is Theorem 2.1.

TheOREM 2.1. Let $p$ and $q$ be two real numbers such that

$$
1<p \leq q<+\infty
$$

Let $\nu$ be a nonnegative measurable function on $] 0,+\infty[$ satisfying (2.2), and $d \mu(t)$ a nonnegative measure on ] $0,+\infty$ [ which satisfies the relation (2.3). Lastly, suppose that

$$
\varphi:] 0,1[\longrightarrow] 0,+\infty[
$$

is a continuous nonincreasing function so that

(i) there exists a positive constant D such that

$$
\forall x, y \in] 0,1[, \quad \varphi(x y) \leq D(\varphi(x)+\varphi(y))
$$

(ii) for all $a>0$,

$$
\int_{0}^{a} \varphi\left(\frac{t}{a}\right) \nu(t) d t<+\infty
$$

Then the following assertions are equivalent.

(1) There exists a positive constant $C_{p, q}$ such that for every nonnegative measurable function $f$,

$$
\left(\int_{0}^{\infty}\left(T_{\varphi}(f)(t)\right)^{q} d \mu(t)\right)^{1 / q} \leq C_{p, q}\left(\int_{0}^{\infty}(f(t))^{p} v(t) d t\right)^{1 / p}
$$

(2) The functions

$$
\begin{aligned}
& r \longmapsto\left(\int_{r}^{\infty} d \mu(t)\right)^{1 / q}\left(\int_{0}^{r} \varphi\left(\frac{t}{r}\right)^{p^{\prime}} \nu(t) d t\right)^{1 / p^{\prime}}, \\
& r \longmapsto\left(\int_{r}^{\infty} \varphi\left(\frac{r}{t}\right)^{q} d \mu(t)\right)^{1 / q}\left(\int_{0}^{r} \nu(t) d t\right)^{1 / p^{\prime}}
\end{aligned}
$$

are bounded on $] 0,+\infty[$, where

$$
p^{\prime}=\frac{p}{p-1}
$$

The proof of this theorem uses the idea of $[10,13,14,18]$ and is left to the reader.

To obtain similar inequalities for the dual operator $T_{\varphi}^{*}$, we use the following duality lemma.

Lemma $2.2[12,18]$. Let $p, q, p^{\prime}, q^{\prime}$ be real numbers such that

$$
1<p \leq q<+\infty, \quad \frac{1}{p}+\frac{1}{p^{\prime}}=1, \quad \frac{1}{q}+\frac{1}{q^{\prime}}=1
$$


let $\mu$ be a $\sigma$-finite measure on ] $0,+\infty$ [ and $v$ a nonnegative locally integrable function on ] $0,+\infty[$. Then the following statements are equivalent.

(1) There exists a positive constant $C_{p, q}$ such that for every nonnegative measurable function $f$

$$
\left(\int_{0}^{\infty}\left(T_{\varphi}(f)(t)\right)^{q} d \mu(t)\right)^{1 / q} \leq C_{p, q}\left(\int_{0}^{\infty}(f(t))^{p} v(t) d t\right)^{1 / p} .
$$

(2) There exists a positive constant $C_{p, q}$ such that for every nonnegative measurable function $g$

$$
\left(\int_{0}^{\infty}\left(T_{\varphi}^{*}(g)(t)\right)^{p^{\prime}} v(t) d t\right)^{1 / p^{\prime}} \leq C_{p, q}\left(\int_{0}^{\infty}(g(t))^{q^{\prime}} d \mu(t)\right)^{1 / q^{\prime}} .
$$

A consequence of Theorem 2.1 and Lemma 2.2 is the following.

THeOREm 2.3 (dual theorem). Under the hypothesis of Theorem 2.1, the following assumptions are equivalent.

(1) There exists a positive constant $C_{p, q}$ such that for every nonnegative measurable function $g$

$$
\left(\int_{0}^{\infty}\left(T_{\varphi}^{*}(g)(x)\right)^{q} \nu(x) d x\right)^{1 / q} \leq C_{p, q}\left(\int_{0}^{\infty}(g(x))^{p} d \mu(x)\right)^{1 / p} .
$$

(2) Both functions

$$
\begin{aligned}
& r \longmapsto\left(\int_{r}^{\infty} d \mu(x)\right)^{1 / p^{\prime}}\left(\int_{0}^{r} \varphi\left(\frac{t}{r}\right)^{q} \nu(t) d t\right)^{1 / q} \\
& r \longmapsto\left(\int_{r}^{\infty} \varphi\left(\frac{r}{x}\right)^{p^{\prime}} d \mu(x)\right)^{1 / p^{\prime}}\left(\int_{0}^{r} \nu(t) d t\right)^{1 / q}
\end{aligned}
$$

are bounded on $] 0,+\infty[$.

\section{Integral operator $T_{\varphi}$ and its dual when the function $\varphi$ is nondecreasing}

In this section, we suppose only that the function

$$
\varphi:] 0,1[\longrightarrow] 0,+\infty[
$$

is nondecreasing, we will give a sufficient condition, which permits to prove that the integral operators $T_{\varphi}$ and $T_{\varphi}^{*}$ satisfy the Hardy inequalities $[1,8,15,16]$.

TheORem 3.1. Let $p$ and $q$ be two real numbers such that

$$
1<p \leq q<\infty
$$

and $p^{\prime}=p /(p-1), q^{\prime}=q /(q-1)$. Let $v$ be a nonnegative function on $] 0,+\infty[$ satisfying (2.2), and $d \mu(t)$ a nonnegative measure on $] 0,+\infty$ [ which satisfies the relation (2.3). Finally, 
let

$$
\varphi:] 0,1[\longrightarrow] 0,+\infty[
$$

be a measurable nondecreasing function.

If there exists $\beta \in[0,1]$ such that the function

$$
r \longmapsto\left(\int_{r}^{\infty} \varphi\left(\frac{r}{x}\right)^{\beta q} d \mu(x)\right)^{1 / q}\left(\int_{0}^{r} \varphi\left(\frac{x}{r}\right)^{p^{\prime}(1-\beta)} v(x) d x\right)^{1 / p^{\prime}}
$$

is bounded on $] 0,+\infty\left[\right.$, then there exists a positive constant $C_{p, q}$ such that for every nonnegative measurable function $f$,

$$
\left(\int_{0}^{\infty}\left(T_{\varphi}(f)(x)\right)^{q} d \mu(x)\right)^{1 / q} \leq C_{p, q}\left(\int_{0}^{\infty}(f(x))^{p} \nu(x) d x\right)^{1 / p} .
$$

Proof of Theorem 3.1. Let $h$ be the function defined by

$$
h(y)=\left(\int_{0}^{y} \varphi\left(\frac{z}{y}\right)^{(1-\beta) p^{\prime}} \nu(z) d z\right)^{1 /\left(p^{\prime}+q\right)} .
$$

By Hölder's inequality, we have

$$
\begin{aligned}
T_{\varphi}(f)(x)= & \int_{0}^{x} \varphi\left(\frac{y}{x}\right) f(y) \nu(y) d y \\
\leq & \left(\int_{0}^{x}\left(\varphi\left(\frac{y}{x}\right)^{\beta} h(y) f(y)\right)^{p} \nu(y) d y\right)^{1 / p} \\
& \times\left(\int_{0}^{x}\left(\varphi\left(\frac{y}{x}\right)^{\beta-1} h(y)\right)^{-p^{\prime}} \nu(y) d y\right)^{1 / p^{\prime}} .
\end{aligned}
$$

Let

$$
J(x)=\int_{0}^{x}\left(\varphi\left(\frac{y}{x}\right)^{\beta-1} h(y)\right)^{-p^{\prime}} \nu(y) d y .
$$

If we replace $h(y)$ by its value, then we obtain

$$
J(x)=\int_{0}^{x} \varphi\left(\frac{y}{x}\right)^{p^{\prime}(1-\beta)}\left(\int_{0}^{y} \varphi\left(\frac{z}{y}\right)^{(1-\beta) p^{\prime}} v(z) d z\right)^{-p^{\prime} /\left(p^{\prime}+q\right)} v(y) d y .
$$

Since the function $\varphi$ is nondecreasing and $\beta \in[0,1]$, we have

$$
\forall 0<y<x, \quad\left(\int_{0}^{y} \varphi\left(\frac{z}{y}\right)^{(1-\beta) p^{\prime}} v(z) d z\right)^{-p^{\prime} /\left(p^{\prime}+q\right)} \leq\left(\int_{0}^{y} \varphi\left(\frac{z}{x}\right)^{(1-\beta) p^{\prime}} v(z) d z\right)^{-p^{\prime} /\left(p^{\prime}+q\right)} .
$$

Therefore

$$
J(x) \leq \int_{0}^{x} \varphi\left(\frac{y}{x}\right)^{(1-\beta) p^{\prime}}\left(\int_{0}^{y} \varphi\left(\frac{z}{x}\right)^{(1-\beta) p^{\prime}} \nu(z) d z\right)^{-p^{\prime} /\left(p^{\prime}+q\right)} v(y) d y
$$


and if we put

$$
g_{x}(z)=\varphi\left(\frac{z}{x}\right)^{(1-\beta) p^{\prime}} 1_{] 0, x[}(z)
$$

from the hypothesis, the function

$$
z \longrightarrow g_{x}(z) v(z)
$$

belongs to $L^{1}(] 0,+\infty[, d z)$ and

$$
J(x) \leq \int_{0}^{x}\left(\int_{0}^{y} g_{x}(z) \nu(z) d z\right)^{-p^{\prime} /\left(p^{\prime}+q\right)} g_{x}(y) v(y) d y .
$$

Since

$$
1-\frac{p^{\prime}}{p^{\prime}+q}=\frac{q}{p^{\prime}+q}>0
$$

then, from [16, Lemma 1], we deduce that

$$
\int_{0}^{x}\left(\int_{0}^{y} g_{x}(z) \nu(z) d z\right)^{-p^{\prime} /\left(p^{\prime}+q\right)} g_{x}(y) \nu(y) d y=\left(\frac{p^{\prime}+q}{q}\right)\left(\int_{0}^{x} g_{x}(z) \nu(z) d z\right)^{q /\left(p^{\prime}+q\right)} ;
$$

therefore inequality (3.14) involves

$$
J(x) \leq\left(\frac{p^{\prime}+q}{q}\right) h(x)^{q} .
$$

From inequalities (3.7) and (3.17), we obtain

$$
\begin{aligned}
\left(T_{\varphi}(f)(x)\right)^{q} & \leq\left(\int_{0}^{x}\left(\varphi\left(\frac{y}{x}\right)^{\beta} h(y) f(y)\right)^{p} v(y) d y\right)^{q / p} J(x)^{q / p^{\prime}} \\
& \leq\left(\frac{p^{\prime}+q}{q}\right)^{q / p^{\prime}}\left(\int_{0}^{x}\left(\varphi\left(\frac{y}{x}\right)^{\beta} h(y) f(y)\right)^{p} v(y) d y\right)^{q / p} h(x)^{q^{2} / p^{\prime}}
\end{aligned}
$$

so, we obtain

$$
\begin{aligned}
I & =\left(\int_{0}^{\infty}\left(T_{\varphi}(f)(x)\right)^{q} d \mu(x)\right)^{1 / q} \\
& \leq\left(\frac{p^{\prime}+q}{q}\right)^{1 / p^{\prime}}\left(\int_{0}^{\infty}\left[\int_{0}^{x}\left(\varphi\left(\frac{y}{x}\right)^{\beta} h(y) f(y) h(x)^{q / p^{\prime}}\right)^{p} \nu(y) d y\right]^{q / p} d \mu(x)\right)^{1 / q} .
\end{aligned}
$$

Since $q / p \geq 1$, then, from Minkowski's inequality [17], we deduce that

$$
I \leq\left(\frac{p^{\prime}+q}{q}\right)^{1 / p^{\prime}}\left(\int_{0}^{\infty}\left(\int_{y}^{\infty}\left(\varphi\left(\frac{y}{x}\right)^{\beta} h(y) f(y) h(x)^{q / p^{\prime}}\right)^{q} d \mu(x)\right)^{p / q} \nu(y) d y\right)^{1 / p}
$$


So

$$
\begin{aligned}
I \leq & \left(\frac{p^{\prime}+q}{q}\right)^{1 / p^{\prime}} \\
& \times\left(\int_{0}^{\infty}\left(\int_{y}^{\infty}\left(\varphi\left(\frac{y}{x}\right)^{\beta} h(x)^{q / p^{\prime}}\right)^{q} d \mu(x)\right)^{p / q}(h(y) f(y))^{p} \nu(y) d y\right)^{1 / p} .
\end{aligned}
$$

On the other hand, from the hypothesis, the function

$$
r \longrightarrow\left(\int_{r}^{\infty} \varphi\left(\frac{r}{x}\right)^{\beta q} d \mu(x)\right)^{1 / q}\left(\int_{0}^{r} \varphi\left(\frac{x}{r}\right)^{p^{\prime}(1-\beta)} \nu(x) d x\right)^{1 / p^{\prime}}
$$

is bounded on $] 0,+\infty[$, we denote

$$
M_{p, q}=\sup _{r>0}\left(\int_{r}^{\infty} \varphi\left(\frac{r}{x}\right)^{\beta q} d \mu(x)\right)^{1 / q}\left(\int_{0}^{r} \varphi\left(\frac{x}{r}\right)^{p^{\prime}(1-\beta)} v(x) d x\right)^{1 / p^{\prime}}
$$

then

$$
\left(\int_{0}^{x} \varphi\left(\frac{z}{x}\right)^{(1-\beta) p^{\prime}} \nu(z) d z\right)^{1 /\left(p^{\prime}+q\right)} \leq M_{p, q}^{p^{\prime} /\left(p^{\prime}+q\right)}\left(\int_{x}^{\infty} \varphi\left(\frac{x}{z}\right)^{\beta q} d \mu(z)\right)^{-p^{\prime} /\left(q\left(p^{\prime}+q\right)\right)}
$$

which means that

$$
h(x) \leq M_{p, q}^{p^{\prime} /\left(p^{\prime}+q\right)}\left(\int_{x}^{\infty} \varphi\left(\frac{x}{z}\right)^{\beta q} d \mu(z)\right)^{-p^{\prime} /\left(q\left(p^{\prime}+q\right)\right)} .
$$

From inequalities (3.21) and (3.25) we obtain

$$
\begin{aligned}
I \leq & \left(\frac{p^{\prime}+q}{q}\right)^{1 / p^{\prime}} M_{p, q}^{q /\left(p^{\prime}+q\right)} \\
& \times\left[\int_{0}^{\infty}(f(y) h(y))^{p}\left(\int_{y}^{\infty} \varphi\left(\frac{y}{x}\right)^{\beta q}\left(\int_{x}^{\infty} \varphi\left(\frac{x}{z}\right)^{\beta q} d \mu(z)\right)^{-q /\left(p^{\prime}+q\right)} d \mu(x)\right)^{p / q} \nu(y) d y\right]^{1 / p} .
\end{aligned}
$$

Since $\varphi$ is nondecreasing, we have

$$
\begin{aligned}
I \leq & \left(\frac{p^{\prime}+q}{q}\right)^{1 / p^{\prime}} M_{p, q}^{q /\left(p^{\prime}+q\right)} \\
& \times\left[\int_{0}^{\infty}(f(y) h(y))^{p}\left(\int_{y}^{\infty} \varphi\left(\frac{y}{x}\right)^{\beta q}\left(\int_{x}^{\infty} \varphi\left(\frac{y}{z}\right)^{\beta q} d \mu(z)\right)^{-q /\left(p^{\prime}+q\right)} d \mu(x)\right)^{p / q} \nu(y) d y\right]^{1 / p} .
\end{aligned}
$$


From [16, Lemma 1], we have again

$$
\int_{y}^{\infty} \varphi\left(\frac{y}{x}\right)^{\beta q}\left(\int_{x}^{\infty} \varphi\left(\frac{y}{z}\right)^{\beta q} d \mu(z)\right)^{-q /\left(p^{\prime}+q\right)} d \mu(x)=\frac{p^{\prime}+q}{p^{\prime}}\left(\int_{y}^{\infty} \varphi\left(\frac{y}{z}\right)^{\beta q} d \mu(z)\right)^{p^{\prime} /\left(p^{\prime}+q\right)},
$$

so, we get

$$
\begin{aligned}
I \leq & \left(\frac{p^{\prime}+q}{q}\right)^{1 / p^{\prime}}\left(\frac{p^{\prime}+q}{p^{\prime}}\right)^{1 / q} M_{p, q}^{q /\left(p^{\prime}+q\right)} \\
& \times\left[\int_{0}^{\infty}(f(y) h(y))^{p}\left(\int_{y}^{\infty} \varphi\left(\frac{y}{z}\right)^{\beta q} d \mu(z)\right)^{p p^{\prime} /\left(p^{\prime}+q\right) q} v(y) d y\right]^{1 / p} .
\end{aligned}
$$

On the other hand, from the relation (3.23), we deduce that

$$
\int_{y}^{\infty} \varphi\left(\frac{y}{z}\right)^{\beta q} d \mu(z) \leq M_{p, q}^{q}\left(\int_{0}^{y} \varphi\left(\frac{z}{y}\right)^{p^{\prime}(1-\beta)} v(z) d z\right)^{-q / p^{\prime}}
$$

consequently

$$
\left(\int_{y}^{\infty} \varphi\left(\frac{y}{z}\right)^{\beta q} d \mu(z)\right)^{p p^{\prime} /\left(\left(p^{\prime}+q\right) q\right)} \leq M_{p, q}^{p p^{\prime} / p^{\prime}+q}(h(y))^{-p} .
$$

From inequalities (3.29) and (3.31), we deduce that for every nonnegative measurable function $f$, we have

$$
\left(\int_{0}^{\infty}\left(T_{\varphi}(f)(x)\right)^{q} d \mu(x)\right)^{1 / q} \leq C_{p, q}\left(\int_{0}^{\infty}(f(y))^{p} \nu(y) d y\right)^{1 / p},
$$

where

$$
C_{p, q}=\left(\frac{p^{\prime}+q}{q}\right)^{1 / p^{\prime}}\left(\frac{p^{\prime}+q}{p^{\prime}}\right)^{1 / q} M_{p, q}
$$

and $M_{p, q}$ is the constant given by (3.23).

This completes the proof of Theorem 3.1.

From Lemma 2.2 and Theorem 3.1 we obtain the following result.

Theorem 3.2 (dual theorem). Under the hypothesis of Theorem 3.1 if there exists $\beta \in[0,1]$ such that the function

$$
r \longmapsto\left(\int_{r}^{\infty} \varphi\left(\frac{r}{x}\right)^{\beta p^{\prime}} d \mu(x)\right)^{1 / p^{\prime}}\left(\int_{0}^{r} \varphi\left(\frac{x}{r}\right)^{q(1-\beta)} \nu(x) d x\right)^{1 / q}
$$

is bounded on $] 0,+\infty\left[\right.$, then there exists a positive constant $C_{p, q}$ such that for every nonnegative measurable function $g$

$$
\left(\int_{0}^{\infty}\left(T_{\varphi}^{*}(g)(x)\right)^{q} \nu(x) d x\right)^{1 / q} \leq C_{p, q}\left(\int_{0}^{\infty}(g(x))^{p} d \mu(x)\right)^{1 / p} .
$$




\section{Riemann-Liouville and Weyl transforms associated with Jacobi operator}

The Jacobi operator stated in the introduction has been studied by many authors $[2,6,7$, $19,20]$. In particular, we know that, for every complex number $\lambda$, the differential equation

$$
\begin{gathered}
\Delta_{\alpha, \beta} u(x)=-\lambda^{2} u(x), \\
u(0)=1, \quad u^{\prime}(0)=0
\end{gathered}
$$

admits a unique solution $\varphi_{\lambda}^{\alpha, \beta}(x)$ given by

$$
\varphi_{\lambda}^{\alpha, \beta}(x)=F\left(\frac{1}{2}(\rho+i \lambda), \frac{1}{2}(\rho-i \lambda) ; \alpha+1 ;-\sinh ^{2}(x)\right)
$$

where $F$ is the Gaussian hypergeometric function $[9,11]$. Furthermore the function $\varphi_{\lambda}^{\alpha, \beta}$ has the following Mehler integral representation:

$$
\forall x>0, \quad \varphi_{\lambda}^{\alpha, \beta}(x)=\int_{0}^{x} k_{\alpha, \beta}(x, y) \cos (\lambda y) d y
$$

where $k_{\alpha, \beta}$ is the nonnegative kernel given by the relation (1.3).

Many properties of harmonic analysis associated with the operator $\Delta_{\alpha, \beta}$ have been studied and established (convolution product, Fourier-transform, inversion formula, Plancherel and Paley-Wiener theorems).

On the other hand, the following integral transforms are defined for the Jacobi operator.

Definition 4.1. (1) The Riemann-Liouville transform associated with Jacobi operator is the integral transform defined, for every nonnegative measurable function $f$, by

$$
R_{\alpha, \beta}(f)(x)=\int_{0}^{x} k_{\alpha, \beta}(x, y) f(y) d y .
$$

(2) The Weyl transform associated with Jacobi operator is defined, for every nonnegative measurable function $f$, by

$$
W_{\alpha, \beta}(f)(x)=\int_{x}^{\infty} k_{\alpha, \beta}(y, x) f(y) A_{\alpha, \beta}(y) d y,
$$

where $k_{\alpha, \beta}$ is the kernel given by the relation (1.3).

Those integral operators are linked by the following duality relation: for all nonnegative measurable functions $f$ and $g$,

$$
\int_{0}^{\infty} R_{\alpha, \beta}(f)(x) g(x) A_{\alpha, \beta}(x) d x=\int_{0}^{\infty} W_{\alpha, \beta}(g)(x) f(x) d x .
$$

As mentioned in the introduction, those integral transforms have been studied on spaces of regular functions.

Our purpose in this section is to study those operators on the spaces $L^{p}([0, \infty[$, $\left.A_{\alpha, \beta}(x) d x\right), 1<p<\infty$. 
Theorem 4.2. For $-1 / 2<\beta \leq \alpha, \alpha \geq 1 / 2$, and $p>2 \alpha+2$, there exists a positive constant $C_{p, \alpha, \beta}$ such that

(1) for all $f \in L^{p}\left(\left[0, \infty\left[, A_{\alpha, \beta}(x) d x\right)\right.\right.$,

$$
\left\|R_{\alpha, \beta}(f)\right\|_{p, \alpha, \beta} \leq C_{p, \alpha, \beta}\|f\|_{p, \alpha, \beta},
$$

(2) for all $g \in L^{p^{\prime}}\left(\left[0, \infty\left[, A_{\alpha, \beta}(x) d x\right)\right.\right.$,

$$
\left\|\frac{1}{A_{\alpha, \beta}(x)} W_{\alpha, \beta}(g)\right\|_{p^{\prime}, \alpha, \beta} \leq C_{p, \alpha, \beta}\|g\|_{p^{\prime}, \alpha, \beta}
$$

where

$$
p^{\prime}=\frac{p}{p-1}
$$

The proof of this theorem needs the following lemma.

Lemma 4.3. For $\alpha \geq 1 / 2$ and $-1 / 2<\beta \leq \alpha$, there exists a positive constant $a_{\alpha, \beta}$ such that

$$
\forall x>y>0, \quad 0 \leq k_{\alpha, \beta}(x, y) \leq a_{\alpha, \beta}(x-y)^{\alpha-1 / 2} \frac{1}{\sqrt{A_{\alpha, \beta}(x)}} .
$$

Proof of Lemma 4.3. (i) It is clear that $k_{\alpha, \beta}(x, y) \geq 0$.

(ii) From mean value's theorem we deduce that

$$
(\cosh (2 x)-\cosh (2 y))^{\alpha-1 / 2} \leq 2^{\alpha-1 / 2}(x-y)^{\alpha-1 / 2} \sinh ^{\alpha-1 / 2}(2 x) .
$$

Therefore from the relation (1.3) and the facts that $\beta \leq \alpha$ and

$$
\sinh (2 x)=2 \sinh (x) \cosh (x),
$$

we have

$$
k_{\alpha, \beta}(x, y) \leq 2^{\alpha+1 / 2} M_{\alpha, \beta} \Gamma(\alpha+1) \sinh ^{-\alpha-1 / 2}(x) \cosh ^{-\beta-1 / 2}(x) \frac{(x-y)^{\alpha-1 / 2}}{\Gamma(\alpha+1 / 2) \sqrt{\pi}},
$$

where

$$
M_{\alpha, \beta}=\max _{0 \leq t \leq 1 / 2}\left|F\left(\alpha+\beta, \alpha-\beta ; \alpha+\frac{1}{2}, t\right)\right|
$$

hence

$$
0 \leq k_{\alpha, \beta}(x, y) \leq 2^{2 \alpha+\beta+3 / 2} M_{\alpha, \beta} \Gamma(\alpha+1) \frac{(x-y)^{\alpha-1 / 2}}{\sqrt{A_{\alpha, \beta}(x)} \Gamma(\alpha+1 / 2) \sqrt{\pi}} .
$$

We obtain the result by setting

$$
a_{\alpha, \beta}=\frac{2^{2 \alpha+\beta+3 / 2} M_{\alpha, \beta} \Gamma(\alpha+1)}{\Gamma(\alpha+1 / 2) \sqrt{\pi}} .
$$


Proof of Theorem 4.2. Let $T_{\varphi}$ and $T_{\varphi}^{*}$ be the Hardy-type operators defined, respectively, by

$$
\begin{aligned}
& T_{\varphi}(f)(x)=\int_{0}^{x} \varphi\left(\frac{t}{x}\right) f(t) \nu(t) d t \\
& T_{\varphi}^{*}(f)(x)=\int_{x}^{\infty} \varphi\left(\frac{x}{t}\right) f(t) d \mu(t),
\end{aligned}
$$

where

$$
\begin{aligned}
\varphi(t) & =(1-t)^{\alpha-1 / 2}, \\
\nu(t) & =A_{\alpha, \beta}^{1-p^{\prime}}(t), \\
d \mu(t) & =t^{p(\alpha-1 / 2)} A_{\alpha, \beta}^{1-p / 2}(t) d t .
\end{aligned}
$$

(i) Since $\alpha \geq 1 / 2$, the function $\varphi$ is continuous and nonincreasing on $] 0,1[$. Furthermore for all $a, b \in] 0,1[$ we have

$$
1-a b \leq(1-a)+(1-b),
$$

then by using the inequality

$$
(u+v)^{p} \leq \max \left(1,2^{p-1}\right)\left(u^{p}+v^{p}\right), \quad u, v \geq 0,
$$

we deduce that

$$
(1-a b)^{\alpha-1 / 2} \leq D\left((1-a)^{\alpha-1 / 2}+(1-b)^{\alpha-1 / 2}\right),
$$

where $D=\max \left(1,2^{\alpha-3 / 2}\right)$. That is,

$$
\varphi(a b) \leq D(\varphi(a)+\varphi(b)) .
$$

(ii) The function $v$ is locally integrable on $[0,+\infty[$. In fact we have

$$
v(t)=A_{\alpha, \beta}^{1-p^{\prime}}(t) \simeq 2^{2 \rho\left(1-p^{\prime}\right)} t^{(2 \alpha+1)\left(1-p^{\prime}\right)} \quad(t \longrightarrow 0) .
$$

Since $p>2 \alpha+2$, then for all $a>0$,

$$
\begin{gathered}
\int_{0}^{a} \nu(t) d t<\infty, \\
\int_{0}^{a} \varphi\left(\frac{t}{a}\right) \nu(t) d t \leq \int_{0}^{a} \nu(t) d t<\infty .
\end{gathered}
$$

(iii) It is clear that the function

$$
t \rightarrow t^{p(\alpha-1 / 2)} A_{\alpha, \beta}^{1-p / 2}(t)
$$

is continuous on $] 0, \infty$ [. Consequently the measure $d \mu(t)$ is locally finite on $] 0, \infty[$. 
(1) Now we will prove that the operator $T_{\varphi}$ defined latterly satisfies the sufficient condition of Theorem 2.1. Then we must show that the functions

$$
\begin{aligned}
& F(r)=\left(\int_{r}^{\infty} d \mu(t)\right)^{1 / p}\left(\int_{0}^{r}\left(\varphi\left(\frac{t}{r}\right)\right)^{p^{\prime}} \nu(t) d t\right)^{1 / p^{\prime}}, \\
& G(r)=\left(\int_{r}^{\infty}\left(\varphi\left(\frac{r}{t}\right)\right)^{p} d \mu(t)\right)^{1 / p}\left(\int_{0}^{r} \nu(t) d t\right)^{1 / p^{\prime}}
\end{aligned}
$$

are bounded on $] 0, \infty[$. We put

$$
\begin{gathered}
I(r)=\left(\int_{r}^{\infty} d \mu(t)\right)^{1 / p}=\left(\int_{r}^{\infty} t^{p(\alpha-1 / 2)} A_{\alpha, \beta}^{1-p / 2}(t) d t\right)^{1 / p}, \\
J(r)=\left(\int_{0}^{r} \nu(t) d t\right)^{1 / p^{\prime}} .
\end{gathered}
$$

Since

$$
\forall t \in] 0,1[, \quad \varphi(t) \leq 1,
$$

then

$$
\begin{array}{ll}
\forall r>0, & F(r) \leq I(r) J(r), \\
\forall r>0, & G(r) \leq I(r) J(r) .
\end{array}
$$

Now, we have

$$
\begin{aligned}
t^{p(\alpha-1 / 2)} A_{\alpha, \beta}^{1-p / 2}(t)= & 2^{\rho(2-p)} t^{2 \alpha+1-p} \cosh ^{(2 \beta+1)(t)(1-p / 2)}(t) \\
& \times\left(\frac{t}{\sinh (t)}\right)^{(2 \alpha+1)(p / 2-1)}
\end{aligned}
$$

and since $p>2 \alpha+2>2$, we deduce that

$$
\forall t>0, \quad t^{p(\alpha-1 / 2)} A_{\alpha, \beta}^{1-p / 2}(t) \leq t^{2 \alpha+1-p}
$$

and consequently

$$
\forall r>0, \quad I(r) \leq\left(\frac{1}{p-2 \alpha-2}\right)^{1 / p} r^{(2 \alpha+2-p) / p} .
$$

Furthermore, we have

$$
J(r)=\left(\int_{0}^{r} v(t) d t\right)^{1 / p^{\prime}}=\left(\int_{0}^{r} A_{\alpha, \beta}^{1-p^{\prime}}(t) d t\right)^{1 / p^{\prime}} .
$$

Since

$$
A_{\alpha, \beta}^{1-p^{\prime}}(t)=2^{2 \rho\left(1-p^{\prime}\right)} t^{(2 \alpha+1)\left(1-p^{\prime}\right)}\left(\frac{t}{\sinh (t)}\right)^{(2 \alpha+1)\left(1-p^{\prime}\right)} \cosh ^{(2 \beta+1)\left(1-p^{\prime}\right)}(t),
$$


then

$$
A_{\alpha, \beta}^{1-p^{\prime}}(t) \leq t^{(2 \alpha+1)\left(1-p^{\prime}\right)}
$$

Thus, we deduce that

$$
\forall r>0, \quad J(r) \leq\left(\frac{1}{(2 \alpha+1)\left(p^{\prime}-1\right)+1}\right)^{1 / p^{\prime}} r^{\left((2 \alpha+1)\left(1-p^{\prime}\right)+1\right) / p^{\prime}} .
$$

From the relations (4.32) and (4.36), we obtain

$$
\begin{aligned}
\forall r>0, \quad\left(\int_{r}^{\infty} d \mu(t)\right)^{1 / p}\left(\int_{0}^{r} \nu(t) d t\right)^{1 / p^{\prime}} & =I(r) J(r) \\
& \leq\left(\frac{1}{p-2 \alpha-2}\right)^{1 / p}\left(\frac{1}{(2 \alpha+1)\left(p^{\prime}-1\right)+1}\right)^{1 / p^{\prime}}
\end{aligned}
$$

and from the relations (4.29), it follows that both functions $F$ and $G$ are bounded on ] $0, \infty$ [. Therefore from Theorem 2.1, there exists a positive constant $D_{p, \alpha, \beta}$ such that, for every nonnegative measurable function $g$, we have

$$
\left(\int_{0}^{\infty}\left(T_{\varphi}(g)(x)\right)^{p} d \mu(x)\right)^{1 / p} \leq D_{p, \alpha, \beta}\left(\int_{0}^{\infty}(g(x))^{p} \nu(x) d x\right)^{1 / p} .
$$

Let $T_{\alpha, \beta}$ be the operator defined, for every nonnegative measurable function $f$, by

$$
\forall x>0, \quad T_{\alpha, \beta}(f)(x)=\frac{1}{\sqrt{A_{\alpha, \beta}(x)}} \int_{0}^{x}(x-y)^{\alpha-1 / 2} f(y) d y .
$$

Then the operators $T_{\alpha, \beta}$ and $T_{\varphi}$ are connected by the following relation: for every nonnegative measurable function $f$, we have

$$
\forall x>0, \quad T_{\varphi}(g)(x)=x^{1 / 2-\alpha} \sqrt{A_{\alpha, \beta}(x)} T_{\alpha, \beta}(f)(x),
$$

where

$$
g(x)=f(x)\left(A_{\alpha, \beta}(x)\right)^{p^{\prime}-1} .
$$

So the relation (4.38) implies that

$$
\left(\int_{0}^{\infty}\left(T_{\alpha, \beta}(f)(x)\right)^{p} A_{\alpha, \beta}(x) d x\right)^{1 / p} \leq D_{p, \alpha, \beta}\left(\int_{0}^{\infty}(f(x))^{p} A_{\alpha, \beta}(x) d x\right)^{1 / p}
$$

and from Lemma 4.3 we deduce that there exists a positive constant $C_{p, \alpha, \beta}$ such that for every nonnegative measurable function $f$, we have

$$
\left(\int_{0}^{\infty}\left(R_{\alpha, \beta}(f)(x)\right)^{p} A_{\alpha, \beta}(x) d x\right)^{1 / p} \leq C_{p, \alpha, \beta}\left(\int_{0}^{\infty}(f(x))^{p} A_{\alpha, \beta}(x) d t\right)^{1 / p} .
$$


Now we consider $f \in L^{p}\left(\left[0, \infty\left[, A_{\alpha, \beta}(x) d x\right)\right.\right.$; then from the relation (4.43) we have

$$
\left(\int_{0}^{\infty}\left(R_{\alpha, \beta}(|f|)(x)\right)^{p} A_{\alpha, \beta}(x) d x\right)^{1 / p} \leq C_{p, \alpha, \beta}\left(\int_{0}^{\infty}(|f(x)|)^{p} A_{\alpha, \beta}(x) d x\right)^{1 / p}<\infty .
$$

Hence the function

$$
x \longrightarrow R_{\alpha, \beta}(|f|)(x)
$$

is finite almost everywhere. Then the function

$$
x \longrightarrow R_{\alpha, \beta}(f)(x)
$$

is defined almost everywhere, and

$$
\left|R_{\alpha, \beta}(f)(x)\right| \leq R_{\alpha, \beta}(|f|)(x)
$$

therefore

$$
\left(\int_{0}^{\infty}\left|R_{\alpha, \beta}(f)(x)\right|^{p} A_{\alpha, \beta}(x) d x\right)^{1 / p} \leq C_{p, \alpha, \beta}\left(\int_{0}^{\infty}(|f(x)|)^{p} A_{\alpha, \beta}(x) d x\right)^{1 / p} .
$$

This completes the proof of Theorem 4.2(1).

(2) From Theorem 2.3, we deduce that there exists a positive constant $D_{p, \alpha, \beta}$ such that for every nonnegative measurable function $h$, we have

$$
\left(\int_{0}^{\infty}\left(T_{\varphi}^{*}(h)(x)\right)^{p^{\prime}} \nu(x) d x\right)^{1 / p^{\prime}} \leq D_{p, \alpha, \beta}\left(\int_{0}^{\infty}(h(x))^{p^{\prime}} d \mu(x)\right)^{1 / p^{\prime}} .
$$

Let $g$ be a nonnegative measurable function, by setting

$$
h(t)=t^{(p-1)(1 / 2-\alpha)} A_{\alpha, \beta}^{(1 / 2)(p-1)}(t) g(t)
$$

and using the inequality (4.49), we deduce that

$$
\left(\int_{0}^{\infty}\left(\frac{1}{A_{\alpha, \beta}(x)} T_{\alpha, \beta}^{*}(g)(x)\right)^{p^{\prime}} A_{\alpha, \beta}(x) d x\right)^{1 / p^{\prime}} \leq D_{p, \alpha, \beta}\left(\int_{0}^{\infty}(g(x))^{p^{\prime}} A_{\alpha, \beta}(x) d x\right)^{1 / p^{\prime}},
$$

where

$$
T_{\alpha, \beta}^{*}(g)(x)=\int_{x}^{\infty}(t-x)^{\alpha-1 / 2} g(t) A_{\alpha, \beta}^{1 / 2}(t) d t
$$

is the dual operator of $T_{\alpha, \beta}$.

Furthermore for every nonnegative measurable function $g$, we have

$$
W_{\alpha, \beta}(g)(x) \leq a_{\alpha, \beta} T_{\alpha, \beta}^{*}(g)(x),
$$


where $a_{\alpha, \beta}$ is the constant given by Lemma 4.3. Hence both inequalities (4.51) and (4.53) involve that there exists a positive constant $C_{p, \alpha, \beta}$ such that, for every nonnegative measurable function $g$, we have

$$
\left(\int_{0}^{\infty}\left(\frac{1}{A_{\alpha, \beta}(x)} W_{\alpha, \beta}(g)(x)\right)^{p^{\prime}} A_{\alpha, \beta}(x) d x\right)^{1 / p^{\prime}} \leq C_{p, \alpha, \beta}\left(\int_{0}^{\infty}(g(x))^{p^{\prime}} A_{\alpha, \beta}(x) d x\right)^{1 / p^{\prime}} .
$$

For $g \in L^{p^{\prime}}\left(\left[0, \infty\left[, A_{\alpha, \beta}(x) d x\right)\right.\right.$, we complete the proof as the first assertion.

Theorem 4.4. For $-1 / 2<\beta \leq \alpha<1 / 2, \alpha+\beta>0$, and $p>\max (2 \alpha+2,(4 \alpha+4 \beta+4) /(4 \alpha+$ $2 \beta+1)$ ), there exists a positive constant $C_{p, \alpha, \beta}$ such that

(1) for every function $f \in L^{p}(] 0, \infty\left[, A_{\alpha, \beta}(x) d x\right)$,

$$
\left\|R_{\alpha, \beta}(f)\right\|_{p, \alpha, \beta} \leq C_{p, \alpha, \beta}\|f\|_{p, \alpha, \beta},
$$

(2) for every function $g \in L^{p^{\prime}}(] 0, \infty\left[, A_{\alpha, \beta}(x) d x\right)$,

$$
\left\|\frac{1}{A_{\alpha, \beta}(x)} W_{\alpha, \beta}(g)\right\|_{p^{\prime}, \alpha, \beta} \leq C_{p, \alpha, \beta}\|g\|_{p^{\prime}, \alpha, \beta},
$$

where

$$
p^{\prime}=\frac{p}{p-1} .
$$

The proof of this theorem needs the following lemma.

Lemma 4.5. For all $-1 / 2<\beta \leq \alpha<1 / 2$, and $\alpha+\beta>0$,

$$
\forall x>y>0, \quad 0 \leq k_{\alpha, \beta}(x, y) \leq b_{\alpha, \beta}(x-y)^{\alpha-1 / 2} \frac{1}{\cosh ^{\alpha+\beta}(x) \sinh ^{\alpha+1 / 2}(x)},
$$

where

$$
\begin{aligned}
b_{\alpha, \beta} & =2 M_{\alpha, \beta} \frac{\Gamma(\alpha+1)}{\Gamma(\alpha+1 / 2) \sqrt{\pi}}, \\
M_{\alpha, \beta} & =\max _{0 \leq t \leq 1 / 2}\left|F\left(\alpha+\beta, \alpha-\beta ; \alpha+\frac{1}{2} ; t\right)\right| .
\end{aligned}
$$

Proof of Lemma 4.5. from relation (1.3) and the fact that $\beta \leq \alpha$ we deduce that

$$
\begin{aligned}
\forall x>y>0, \quad 0 \leq & k_{\alpha, \beta}(x, y) \\
\leq & \frac{2^{-\alpha+3 / 2} M_{\alpha, \beta} \Gamma(\alpha+1)}{\Gamma(\alpha+1 / 2) \sqrt{\pi}} \\
& \times(\cosh 2 x-\cosh 2 y)^{\alpha-1 / 2} \frac{1}{\cosh ^{\alpha+\beta}(x) \sinh ^{2 \alpha}(x)} .
\end{aligned}
$$


On the other hand

$$
\begin{aligned}
\cosh (2 x)-\cosh (2 y) & =2(\sinh (x)-\sinh (y))(\sinh (x)+\sinh (y)) \\
& \geq 2 \sinh (x)(\sinh (x)-\sinh (y)) .
\end{aligned}
$$

From mean value's theorem and the fact that $\alpha<1 / 2$, we have

$$
(\sinh (x)-\sinh (y))^{\alpha-1 / 2} \leq(x-y)^{\alpha-1 / 2}
$$

by using the inequalities (4.60), (4.61), and (4.62) we deduce that

$$
\begin{aligned}
k_{\alpha, \beta}(x, y) & \leq 2 M_{\alpha, \beta} \frac{\Gamma(\alpha+1)}{\Gamma(\alpha+1 / 2) \sqrt{\pi}}(x-y)^{\alpha-1 / 2} \frac{1}{\cosh ^{\alpha+\beta}(x) \sinh ^{\alpha+1 / 2}(x)} \\
& =b_{\alpha, \beta}(x-y)^{\alpha-1 / 2} \frac{1}{\cosh ^{\alpha+\beta}(x) \sinh ^{\alpha+1 / 2}(x)}
\end{aligned}
$$

which proves the Lemma 4.5 .

Proof of Theorem 4.4. In this proof, we consider the functions $\varphi, \nu$ and the measure $d \mu$ defined, respectively, by

$$
\begin{gathered}
\varphi(t)=(1-t)^{\alpha-1 / 2}, \\
v(t)=A_{\alpha, \beta}^{1-p^{\prime}}(t), \\
d \mu(t)=t^{p(\alpha-1 / 2)} \sinh ^{2 \alpha+1-p(\alpha+1 / 2)}(t) \cosh ^{2 \beta+1-p(\alpha+\beta)}(t) d t .
\end{gathered}
$$

(i) Since $-1 / 2<\alpha<1 / 2$, the function $\varphi$ is increasing on $] 0,1[$.

(ii) For $p>2 \alpha+2, \int_{0}^{a} v(t) d t<\infty$ for all $a>0$.

(iii) $\int_{a}^{b} d \mu(t)<\infty$ for $0<a<b<\infty$.

We will prove that the operator $T_{\varphi}$ satisfies the sufficient conditions of Theorem 3.1, that is, there exists $\lambda \in[0,1]$ such that the function

$$
F(r)=\left(\int_{r}^{\infty} \varphi\left(\frac{r}{x}\right)^{\lambda p} d \mu(x)\right)^{1 / p}\left(\int_{0}^{r} \varphi\left(\frac{x}{r}\right)^{p^{\prime}(1-\lambda)} \nu(x) d x\right)^{1 / p^{\prime}}
$$

is bounded on $] 0, \infty[$. In fact we denote

$$
\begin{aligned}
& I(r)=\left(\int_{r}^{\infty} \varphi\left(\frac{r}{x}\right)^{\lambda p} d \mu(x)\right)^{1 / p} \\
& J(r)=\left(\int_{0}^{r} \varphi\left(\frac{x}{r}\right)^{p^{\prime}(1-\lambda)} \nu(x) d x\right)^{1 / p^{\prime}}
\end{aligned}
$$

Since $\alpha<1 / 2$ and $p>\max (2 \alpha+2,(4 \alpha+4 \beta+4) /(4 \alpha+2 \beta+1))$, we deduce that

$$
(I(r))^{p} \leq M_{\alpha, \beta, p} \int_{r}^{\infty} h(x) d x
$$


where

$$
\begin{aligned}
& M_{\alpha, \beta, p}= \max \left[\sup _{[0,1]}\left(\left(\frac{\sinh (x)}{x}\right)^{2 \alpha+1-p(\alpha+1 / 2)} \cosh ^{2 \beta+1-p(\alpha+\beta)}(x)\right),\right. \\
&\left.\sup _{[1, \infty[}\left(\cosh ^{2 \beta+1-p(\alpha+\beta)}(x) \sinh ^{2 \alpha+1-p(\alpha+1 / 2)}(x) e^{(p(2 \alpha+\beta+1 / 2)-(2 \alpha+2 \beta+2)) x}\right)\right], \\
& h(x)=\left\{\begin{array}{cc}
\left(1-\frac{r}{x}\right)^{(\alpha-1 / 2) \lambda p} x^{2 \alpha+1-p,} & 0<x \leq 1, \\
\left(1-\frac{r}{x}\right)^{(\alpha-1 / 2) \lambda p} e^{(2 \alpha+2 \beta+2-p(2 \alpha+\beta+1 / 2)) x}, & 1<x .
\end{array}\right.
\end{aligned}
$$

So if we pick $\lambda$ in $] \max (0,(1-p(1 / 2+\alpha)) / p(1 / 2-\alpha)) ; \min (1,1 / p(1 / 2-\alpha))]$, we can prove that there exist $C_{1}(\alpha, \beta, p)>0$ and $C_{2}(\alpha, \beta, p)>0$ such that

$$
\begin{aligned}
& I(r) \leq C_{1}(\alpha, \beta, p) r^{(2 \alpha+2-p) / p}, \\
& J(r) \leq C_{2}(\alpha, \beta, p) r^{\left((2 \alpha+1)\left(1-p^{\prime}\right)+1\right) / p^{\prime}} .
\end{aligned}
$$

This involves that the function $F$ is bounded on ]0, [. Then from Theorems 3.1 and 3.2 , we know that there exists a positive constant $C_{p, \alpha, \beta}^{\prime}$ such that for every nonnegative measurable function $g$, we have

$$
\begin{gathered}
\left(\int_{0}^{\infty}\left(T_{\varphi}(g)(x)\right)^{p} d \mu(x)\right)^{1 / p} \leq C_{p, \alpha, \beta}^{\prime}\left(\int_{0}^{\infty}(g(x))^{p} \nu(x) d t\right)^{1 / p}, \\
\left(\int_{0}^{\infty}\left(T_{\varphi}^{*}(g)(x)\right)^{p^{\prime}} \nu(x) d x\right)^{1 / p^{\prime}} \leq C_{p, \alpha, \beta}^{\prime}\left(\int_{0}^{\infty}(g(x))^{p^{\prime}} d \mu(x)\right)^{1 / p^{\prime}} .
\end{gathered}
$$

Let $K_{\alpha, \beta}$ be the integral operator defined by

$$
\forall x>0, \quad K_{\alpha, \beta}(f)(x)=\frac{1}{\cosh ^{\alpha+\beta}(x) \sinh ^{\alpha+1 / 2}(x)} \int_{0}^{x}(x-y)^{\alpha-1 / 2} f(y) d y
$$

and its dual defined by

$$
\forall x>0, \quad K_{\alpha, \beta}^{*}(f)(x)=\int_{x}^{\infty}(t-x)^{\alpha-1 / 2} f(t) \sinh ^{(1 / 2+\alpha)}(t) \cosh ^{(\beta-\alpha+1)}(t) d t .
$$

The operators $T_{\varphi}$ and $K_{\alpha, \beta}$, respectively, $T_{\varphi}^{*}$ and $K_{\alpha, \beta}^{*}$ are connected by the relation

$$
\forall x>0, \quad T_{\varphi}\left(g_{1}\right)(x)=x^{1 / 2-\alpha} \cosh ^{\alpha+\beta}(x) \sinh ^{\alpha+1 / 2}(x) K_{\alpha, \beta}(f)(x),
$$

where

$$
\forall x>0, \quad g_{1}(x)=f(x)\left(A_{\alpha, \beta}(x)\right)^{p^{\prime}-1},
$$


respectively,

$$
\forall x>0, \quad T_{\varphi}^{*}\left(g_{2}\right)(x)=K_{\alpha, \beta}^{*}(f)(x)
$$

where

$$
g_{2}(x)=f(x) x^{(p-1)(1 / 2-\alpha)} \sinh ^{(p-1)(1 / 2+\alpha)}(x) \cosh ^{(p-1)(\alpha+\beta)}(x) .
$$

The relations (4.70), (4.73), and (4.75) imply that for every nonnegative measurable function $f$, we have

$$
\begin{gathered}
\left(\int_{0}^{\infty}\left(K_{\alpha, \beta}(f)(x)\right)^{p} A_{\alpha, \beta}(x) d x\right)^{1 / p} \leq D_{p, \alpha, \beta}\left(\int_{0}^{\infty}(f(x))^{p} A_{\alpha, \beta}(x) d x\right)^{1 / p}, \\
\left(\int_{0}^{\infty}\left(\frac{1}{A_{\alpha, \beta}(x)} K_{\alpha, \beta}^{*}(f)(x)\right)^{p^{\prime}} A_{\alpha, \beta}(x) d x\right)^{1 / p^{\prime}} \leq D_{p, \alpha, \beta}\left(\int_{0}^{\infty}(f(x))^{p^{\prime}} A_{\alpha, \beta}(x) d x\right)^{1 / p^{\prime}} .
\end{gathered}
$$

We complete the proof of Theorem 4.4 by the same way as Theorem 4.2 and using Lemma 4.5.

\section{References}

[1] K. F. Andersen and H. P. Heinig, Weighted norm inequalities for certain integral operators, SIAM J. Math. Anal. 14 (1983), no. 4, 834-844.

[2] W. C. Connett and A. L. Schwartz, The Littlewood-Paley theory for Jacobi expansions, Trans. Amer. Math. Soc. 251 (1979), 219-234.

[3] L. Debnath, Integral Transforms and Their Applications, CRC Press, Florida, 1995.

[4] Recent applications of fractional calculus to science and engineering, Int. J. Math. Math. Sci. 2003 (2003), no. 54, 3413-3442.

[5] M. Dziri, Inégalités de Hardy-Littlewood pour une classe d'opérateurs intégraux, Diplôme d'études approfondies, Faculté des Sciences de Tunis, département de Mathématiques, July 2001.

[6] M. Flensted-Jensen and T. Koornwinder, The convolution structure for Jacobi function expansions, Ark. Mat. 11 (1973), 245-262.

[7] G. Gasper, Banach algebras for Jacobi series and positivity of a kernel, Ann. of Math. (2) 95 (1972), 261-280.

[8] P. R. Halmos, Measure Theory, Springer-Verlag, New York, 1974.

[9] T. Koornwinder, A new proof of a Paley-Wiener type theorem for the Jacobi transform, Ark. Mat. 13 (1975), 145-159.

[10] Q. Lai, Weighted modular inequalities for Hardy type operators, Proc. London Math. Soc. (3) 79 (1999), no. 3, 649-672.

[11] N. N. Lebedev, Special Functions and Their Applications, Dover Publications, New York, 1972.

[12] F. J. Martín-Reyes and E. Sawyer, Weighted inequalities for Riemann-Liouville fractional integrals of order one and greater, Proc. Amer. Math. Soc. 106 (1989), no. 3, 727-733.

[13] V. G. Maz'ja, Sobolev Spaces, Springer Series in Soviet Mathematics, Springer-Verlag, Berlin, 1985.

[14] B. Muckenhoupt, Hardy's inequality with weights, Studia Math. 44 (1972), 31-38.

[15] G. E. Shilov and B. L. Gurevich, Integral, measure and derivative: a unified approach, Dover Books on Advanced Mathematics, Dover Publications, New York, 1977. 


\section{Hardy-type inequalities}

[16] G. J. Sinnamon, Weighted Hardy and Opial-type inequalities, J. Math. Anal. Appl. 160 (1991), no. $2,434-445$.

[17] E. M. Stein and G. Weiss, Introduction to Fourier Analysis on Euclidean Spaces, Princeton University Press, New Jersey, 1971.

[18] V. D. Stepanov, Weighted inequalities for a class of Volterra convolution operators, J. London Math. Soc. (2) 45 (1992), no. 2, 232-242.

[19] K. Trimèche, Transformation intégrale de Weyl et théorème de Paley-Wiener associés à un opérateur différentiel singulier sur $(0, \infty)$ [Weyl integral transform and Paley-Wiener theorem associated with a singular differential operator on $(0, \infty)]$, J. Math. Pures Appl. (9) 60 (1981), no. 1, 51-98 (French).

[20] - Inversion of the Lions transmutation operators using generalized wavelets, Appl. Comput. Harmon. Anal. 4 (1997), no. 1, 97-112.

M. Dziri: Department of Mathematics, Faculty of Sciences of Tunis, University of Tunis, 1060 Tunis, Tunisia

E-mail address: moncef.dziri@iscae.rnu.tn

L. T. Rachdi: Department of Mathematics, Faculty of Sciences of Tunis, University of Tunis, 1060 Tunis, Tunisia

E-mail address: lakhdartannech.rachdi@fst.rnu.tn 


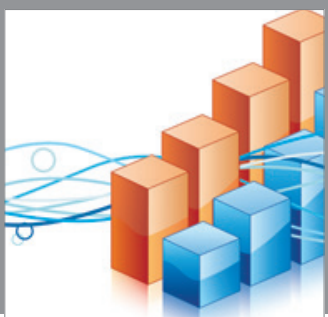

Advances in

Operations Research

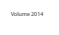

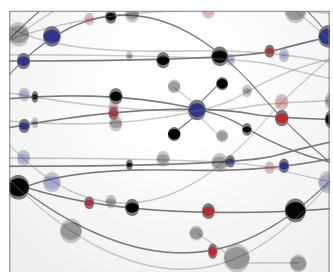

\section{The Scientific} World Journal
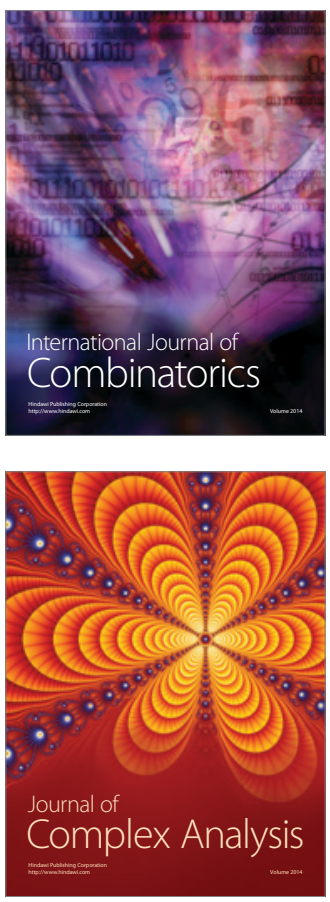

International Journal of

Mathematics and

Mathematical

Sciences
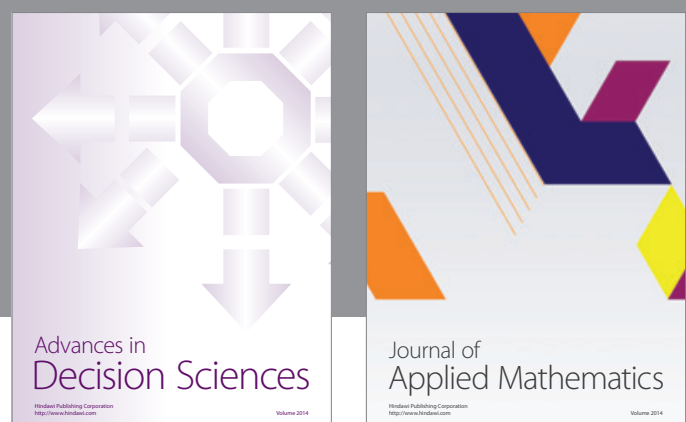

Journal of

Applied Mathematics
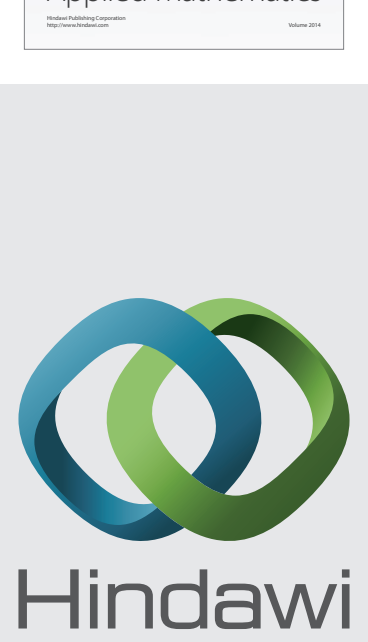

Submit your manuscripts at http://www.hindawi.com
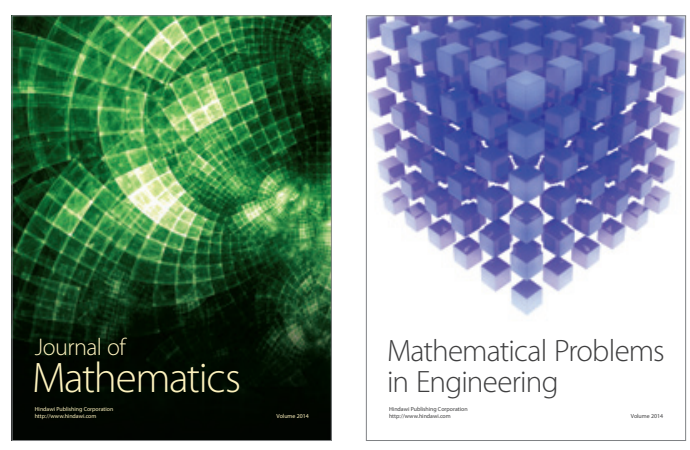

Mathematical Problems in Engineering
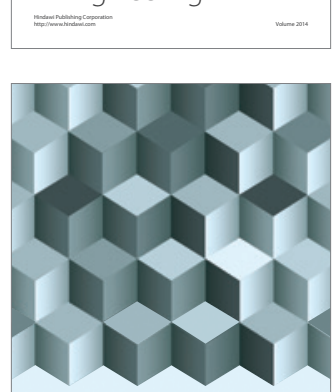

Journal of

Function Spaces
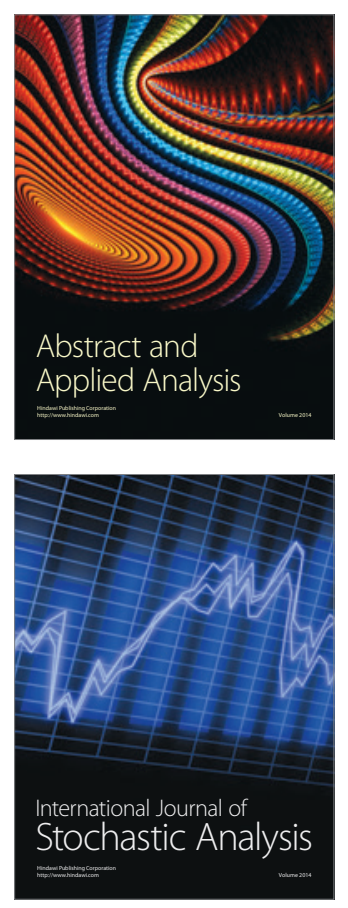

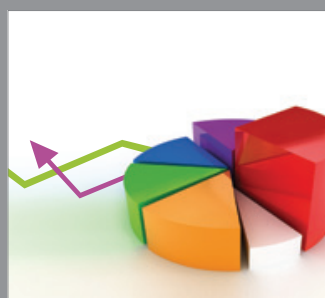

ournal of

Probability and Statistics

Promensencen
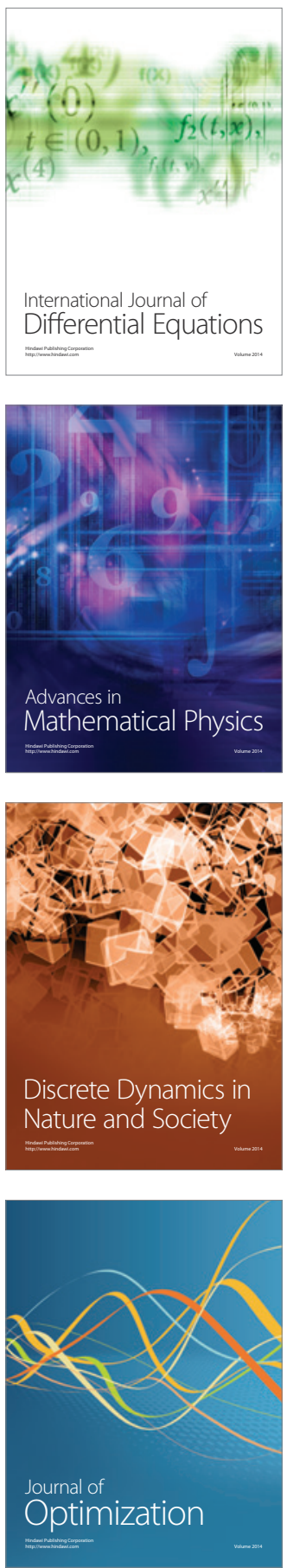